\title{
Logarithmic contribution to the density of states of rectangular Andreev billiards
}

\author{
A. Kormányos, ${ }^{*}$ Z. Kaufmann, and J. Cserti \\ Department of Physics of Complex Systems, Eötvös University, H-1117 Budapest, Pázmány Péter sétány 1/A, Hungary \\ C. J. Lambert \\ Department of Physics, Lancaster University, Lancaster, LA1 4YB, United Kingdom
}

(Received 18 October 2002; published 15 May 2003)

\begin{abstract}
We demonstrate that the exact quantum mechanical calculations are in good agreement with the semiclassical predictions for rectangular Andreev billiards, and therefore for a large number of open channels it is sufficient to investigate the Bohr-Sommerfeld approximation of the density of states. We present exact calculations of the classical path length distribution $P(s)$, which is a nondifferentiable function of $s$, but whose integral is a smooth function with logarithmically dependent asymptotic behavior. Consequently, the density of states of rectangular Andreev billiards has two contributions on the scale of the Thouless energy: one which is well-known and is proportional to the energy, and the other which shows a logarithmic energy dependence. It is shown that the prefactors of both contributions depend on the geometry of the billiards but have universal limiting values when the width of the superconductor tends to zero.
\end{abstract}

DOI: 10.1103/PhysRevB.67.172506 PACS number(s): 74.45.+c, 03.65.Sq, 05.45.Mt, 74.50.+r

When a normal quantum dot is placed in contact with a superconductor, the low-energy density of states $n(E)$ of the resulting Andreev billiard is strongly modified compared with the normal state. ${ }^{1}$ The energy dependence of $n(E)$ is highly nontrivial and provides a testing ground for current understanding of proximity effects of hybrid superconducting nanostructures and for theoretical tools such as semiclassical theory. Recently a number of conflicting results have been obtained for the density of states (DOS) of ballistic Andreev billiards (AB). One example is shown in Fig. 1, which in the separable limit $W=a$ was studied long ago in, ${ }^{2}$ where it was found that for small $E, n(E)$ is proportional to $E$. In contrast for $W<a$, the problem has only recently been studied. ${ }^{1,3,4,6,5}$ In Ref. 3 it was found that $n(E)=\nu E$, where $\nu$ is a universal constant, independent of $E$ and $W$. In contrast, Ref. 4 predicted different values for $\nu$.

In this paper we resolve this discrepancy by showing that neither of these results is strictly correct in the limit $E \rightarrow 0$ and instead predict that $n(E)$ diverges logarithmically. This generic logarithmic contribution is significant for energies less than or of the order the Thouless energy.

To address this problem, we use both exact and semiclassical techniques to analyze the AB of Fig. 1. The exact calculation starts with a ballistic two dimensional normal dot of area $A$, described by a scattering matrix $S_{0}(E)$ and with a

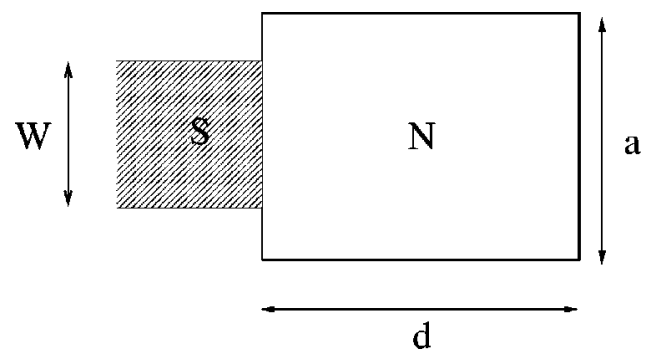

FIG. 1. A normal quantum dot of rectangular shape in contact with a superconductor placed at the middle of the edge of the normal region. mean level spacing for the isolated normal system $\delta$ $=2 \pi \hbar^{2} / m A$ at the Fermi energy $E_{F}$. Then, a superconductor of width $W$ and bulk order parameter $\Delta$ is placed in contact with such a billiard. The number of open channels in the $S$ region is the integer part of $M=k_{F} W / \pi$, and the energy levels of $\mathrm{AB}$ are the positive eigenvalues $E$ (measured from the Fermi energy) of the Bogoliubov-de Gennes equation. ${ }^{7}$ A secular equation of the exact energy levels of $\mathrm{AB}$, in terms of the scattering matrix $S_{0}(E)$ of the normal region, is derived by matching the wave functions at the interface of the normal-superconducting $(N-S)$ systems. The energy levels are exact in the sense that no Andreev approximation $\left[\Delta / E_{F} \ll 1\right.$ and quasiparticles whose incident or reflected directions are approximately perpendicular to the $N-S$ interface $\left.^{8}(\mathrm{BS})\right]$ is assumed.

We also give the semiclassical Bohr-Sommerfeld approximation of the DOS $n(E)$ expressed by the classical return probability $P(s)$ of the electron. Our exact and semiclassical calculations are applied to the integrable $\mathrm{AB}$ shown in Fig. 1. We demonstrate that the integrated DOS agrees very well with the exact calculations. We find that the integral of the return probability $P(s)$ has a contribution depending on $s$ logarithmically in the asymptotic limit, $s \rightarrow \infty$, which has to date been overlooked. As a consequence, the small energy dependence of the DOS $n(E)$ has also a logarithmic factor in addition to a contribution which depends linearly on the energy $E$ as predicted in Refs. 3 and $4 .^{9}$

In our earlier work ${ }^{5}$ we found that the exact energy levels of $\mathrm{AB}$ with arbitrary shape of normal region are the solutions of the following secular equation:

$$
\operatorname{det}\left[\operatorname{Im}\left\{\gamma D_{e}(E) D_{h}(E)\right\}\right]=0
$$

where

$$
\begin{gathered}
D_{e}(E)=Q(E)+K(E) G(E), \\
D_{h}(E)=\left[Q(-E)-K(-E) G^{*}(-E)\right]^{-1},
\end{gathered}
$$




$$
G(E)=\left[1-S_{0}(E)\right]\left[1+S_{0}(E)\right]^{-1} .
$$

Here $\gamma=e^{+i \arccos (E / \Delta)}, Q$ and $K$ are diagonal matrices with elements $Q_{n m}(E)=\delta_{n m} q_{n}(E) \quad$ and $\quad K_{n m}(E)=\delta_{n m} k_{n}(E)$, where $q_{n}(E)=k_{F} \sqrt{1+i \sqrt{\Delta^{2}-E^{2}} / E_{F}-n^{2} / M^{2}}$ are the transverse wave numbers of the electron in the $S$ region, and $k_{n}(E)=k_{F} \sqrt{1+E / E_{F}-n^{2} / M^{2}}$ are the transverse wavenumbers of the electron in the $\mathrm{S}$ region when $\Delta=0$. It is assumed that the Fermi wavenumber, $k_{F}=\sqrt{2 m E_{F} / \hbar^{2}}$, is the same in the $S$ and $N$ regions. All the matrices are $M \times M$ dimensional. All the information on the normal region are incorporated in the matrix $G$ via the scattering matrix $S_{0}(E)$. Note that the secular equation (1a) is an extension of that derived for box and disk geometries in Ref. 6.

The density of states in the semiclassical BohrSommerfeld approximation is written as ${ }^{5}$

$$
\begin{aligned}
n(E)= & M \int_{0}^{\infty} d s P(s)\left[\frac{s}{\hbar v_{F}}+\frac{1}{\sqrt{\Delta^{2}-E^{2}}}\right] \\
& \times \sum_{n=0}^{\infty} \delta\left(\frac{s E}{\hbar v_{F}}-\left(n \pi+\arccos \frac{E}{\Delta}\right)\right) .
\end{aligned}
$$

This expression reduces to that of by Melsen et al., ${ }^{3}$ Schomerus and Beenakker, ${ }^{1}$ Lodder and Nazarov, ${ }^{1}$ and Ihra et al., ${ }^{4}$ in the limit $E \ll \Delta$ and when the coherence length in the superconductor, $\xi_{0}=\hbar v_{\mathrm{F}} / \Delta \ll 2 d$. In that case, the energy dependent phase shift $-\arccos (E / \Delta)$, due to Andreev reflections, was approximated by $\pi / 2$. However, our expression is valid without such an approximation, and essentially improves the agreement between the exact and the BohrSommerfeld approximation of the DOS (see also Ref. 5).

From Eq. (2) one can find a simple expression for the integrated DOS $N(E)=\int_{0}^{E} n\left(E^{\prime}\right) d E^{\prime}$ :

$$
N(E)=M \sum_{n=0}^{\infty}\left\{1-F\left[s_{n}(E)\right]\right\},
$$

where

$$
\begin{gathered}
s_{n}(E)=\frac{(n \pi+\arccos E / \Delta)}{E / \Delta} \xi_{0}, \\
F(s)=\int_{0}^{s} P\left(s^{\prime}\right) d s^{\prime},
\end{gathered}
$$

where $F(s)$ is the integrated distribution function of the return probability of the electron. Note that $P(s)$ is normalized to one, i.e., $F(\infty)=1$.

To obtain the exact energy levels of the AB shown in Fig. 1 , we need to calculate the scattering matrix $S_{0}(E)$ of the $N$ region. Following the same approach as Mortensen and co-workers, ${ }^{10}$ we obtain

$$
S_{0}(E)=\varrho R(X, Q) \varrho^{T}-1,
$$

where

$$
R(X, Q)=2(1+Q)^{-1}\left\{1-2 X^{2}\left[1+X^{2}+Q\left(1-X^{2}\right)\right]^{-1}\right\} .
$$

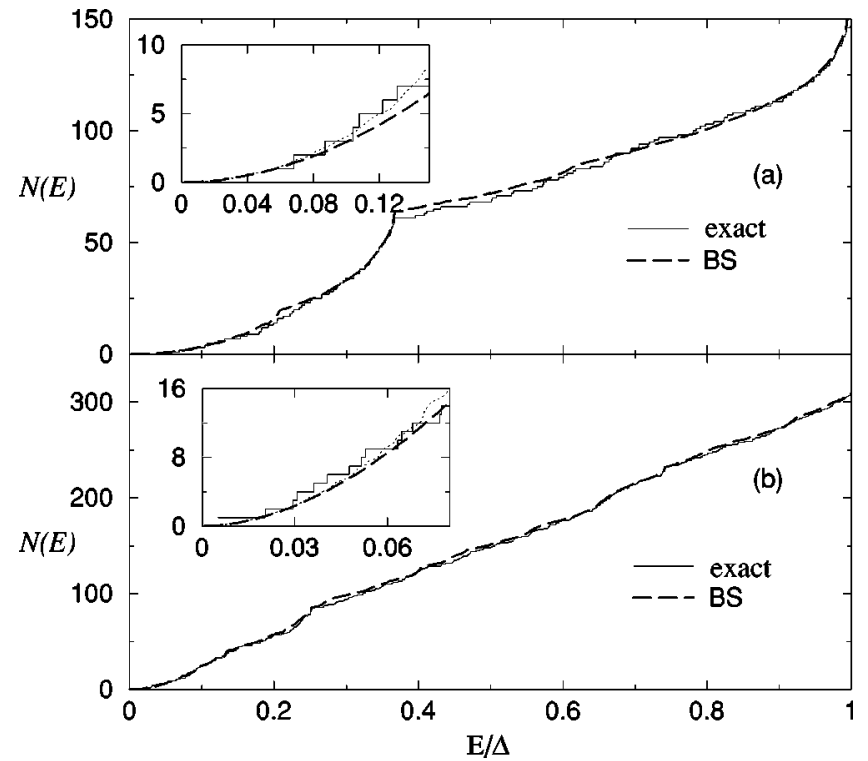

FIG. 2. The integrated density of states $N(E)$ from exact quantum mechanical calculations (solid lines) and from the BohrSommerfeld approximation given by Eq. (3) (dashed lines) as functions of $E$ (in units of $\Delta$ ) for $W=0.8 a$ (a) and $W=0.5 a$ (b). Insets show the enlarged portions of $N(E)$ from exact calculations (solid line), its BS approximation from Eq. (3) (dotted line), and its asymptotic form from Eq. (8a) (dashed line). In both cases $d=a$ and the parameters are $M=55.5, \Delta / E_{F}=0.015$.

Here $X$ is a diagonal matrix with elements $X_{m n}$ $=\delta_{m n} \exp \left(i k_{n} d\right), Q=\varrho^{T} \varrho$, and $\varrho$ is a $\left[k_{\mathrm{F}} W / \pi\right] \times\left[k_{\mathrm{F}} a / \pi\right]$ dimensional matrix with elements given by the overlap integrals defined in Ref. 10 ([ []$^{]}$stands for the integer part). Notice that if $W=a$, then $\varrho=1$ and the scattering matrix $S_{0}(E)=-X^{2}$ is a diagonal matrix. In this case, we obtain the same secular equation for $\mathrm{AB}$ as that in Ref. 6 for box geometries.

In Fig. 2 the exactly (numerically) computed integrated density of states obtained from Eq. (1) and its evaluation in Bohr-Sommerfeld (BS) approximation using Eq. (3) are shown for different widths $W$ of the lead [for the calculation of $P(s)$ see below]. This shows that the exact calculations and the semiclassical predictions are in very good agreement.

Calculations for $P(s)$ and $F(s)$ start with unfolding the trajectory of the electron, i.e., using the fact that the free motion of the particle in the billiard is equivalent to its flight in a lattice of vertical intervals with length $W$ and with lattice constant $2 d(a)$ in the horizontal (vertical) direction. In general, one can calculate $P(s)$ by using a large number of trajectories and determining the distribution of their path lengths. An analytic form of $P(s)$ and $F(s)$ can be derived for $W \geqslant a / 2$. As an example, these functions are plotted for $d=a$ and $W=a / 2$ in Fig. 3. Clearly, for arbitrary $W, P(s)$ $=0$ for $s<2 d$ and for larger $s$ it is a nondifferentiable function possessing a singularity at $s=2 d$ and peaks at multiples of $2 d$. Using Eq. (2) one can calculate the DOS and pronounced peaks (indeed singularities) arise due to the singularity of $P(s)$ at $s=2 d$. The positions of these singularities in the DOS are in perfect agreement with that obtained from the general expression derived in Ref. 6. 

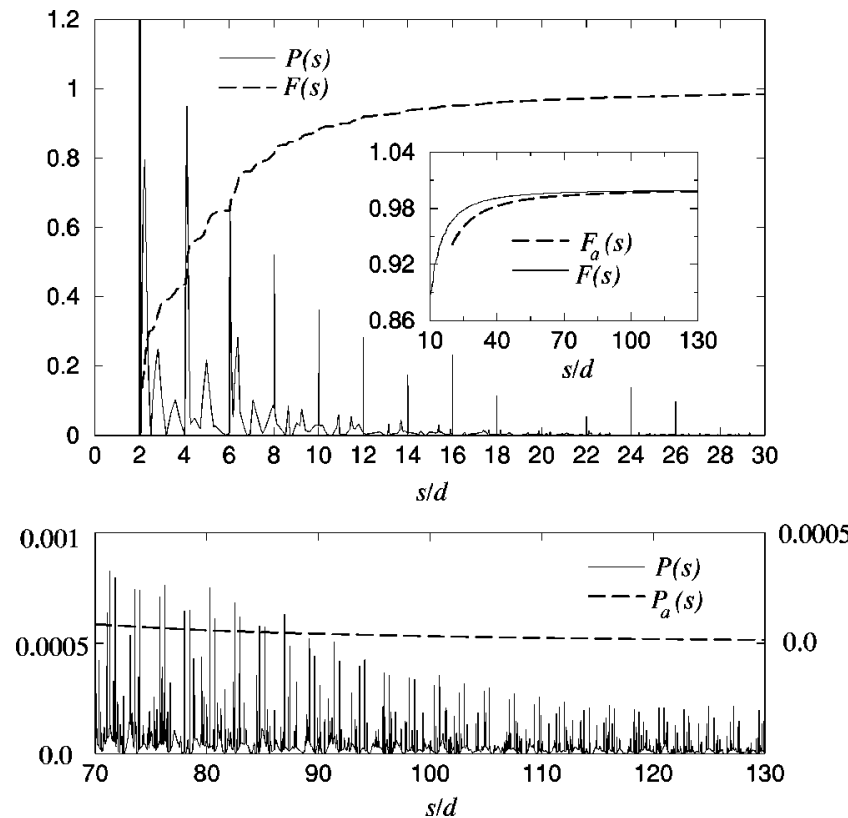

FIG. 3. Top panel: the return probability $P(s)$ (solid line) and its integral $F(s)$ (dashed line) as functions of $s / d$ for $W=a / 2$ and $d$ $=a$. In the inset $F(s)$ (solid line) and its asymptotic form $F_{a}(s)$ given by Eq. (5) (dashed line) are plotted as functions of $s / d$ for large $s$. Bottom panel: $P(s)$ (solid line and left $y$ axis) for larger $s$ along with the asymptotic expression of the return probability $P_{a}(s)=d F_{a}(s) / d s$ (dashed line and right $y$ axis).

The integrated path length distribution $F(s)$ is a smooth function of $s$ allowing one to obtain its asymptotic form $F_{a}(s)$ for $s \rightarrow \infty$ (see the inset of the top panel of Fig. 3). From Eq. (2) one can see that the large $s$ behavior of $P(s)$ is related to the low energy dependence of the DOS. Due to the rapid variation of $P(s)$, it is difficult to define its asymptote. To avoid this problem we first calculate the asymptotic behavior of $F(s)$ and the asymptotic form of $P(s)$ is then calculated from $P_{a}(s)=d F_{a}(s) / d s$ (see also the bottom panel of Fig. 3). For $s \rightarrow \infty$ the analytical form of the asymptote of $F(s)$ is found by considering how particles can travel a large distance $s$ without hitting the vertical intervals of length $W$ in the unfolded space. Such trajectories lie only in certain directions forming corridors with slopes $u$ that are multiples of $a /(2 k d)$, where $k=[W / a]$. The probability that a particle runs in such a corridor is proportional to $1 /\left(u s^{2}\right)$ for large $u$ but $|u|<u_{\max }=s / x_{\min }$, where $x_{\min }$ is a minimal displacement in the horizontal direction. Summation of the probabilities for these corridors yields a term proportional to $s^{-2} \ln u_{\max }$. Directions with slopes larger than $u_{\max }$ should be treated separately and the result contains a term $s^{-2} \ln x_{\min }$. The above terms from the two regions sum up to $s^{-2} \ln s$, and finally we find

$$
F(s) \approx F_{a}(s) \equiv 1-\left(C_{1}+C_{2} \ln \frac{s}{\sqrt{A}}\right) \frac{A}{s^{2}}
$$

for large $s$. Here $C_{1}$ and $C_{2}$ are functions of $d / a$ and $W / a$. For the case $W \geqslant a / 2$ the detailed calculation gives analytic expressions also for $C_{1}$ and $C_{2}$ :

$$
\begin{gathered}
C_{1}=\frac{d}{a}\left(8-6 \frac{W}{a}\right)+C_{2}\left[c-\frac{1}{2} \ln (d / a)\right], \\
C_{2}=\frac{4 d}{W}\left(1-\frac{W}{a}\right)^{2}, \\
c=\gamma+\frac{1}{2}+2 \sum_{j=1}^{\infty} K_{0}\left(4 \pi j \frac{d}{a}\right) .
\end{gathered}
$$

Here $\gamma$ is the Euler-Mascheroni constant and $K_{0}$ is the modified Bessel function of the second kind. Therefore, $c$ is a weakly dependent function of $d$ for $d \geqslant a$.

In the case of $W<a / 2$ additional passages with slopes corresponding to noninteger multiples of $a /(2 d)$ open and $C_{1}, C_{2}$ are modified. $C_{1}$ is numerically determined, while for $C_{2}$ we have the formula

$$
C_{2}=\frac{4 d}{W} \sum_{m=1}^{[a / W]} \frac{N_{m}}{m}\left(1-m \frac{W}{a}\right)^{2},
$$

where $N_{m}$ is the number of those integers in the interval $[0, m-1]$ which are relative prime to $m$. (Note that $\left.N_{1}=1\right)$.

Using the smooth asymptote of $F(s)$ given by Eq. (5) and Eqs. (3), an analytic expression can be found for the low energy behavior of the DOS:

$$
\begin{gathered}
N_{a}(E)=\frac{M}{8 \pi^{2}}\left(\frac{E}{E_{T}}\right)^{2}\left[\alpha+\beta\left(\frac{1}{2}-\ln \frac{E}{E_{T}}\right)\right], \\
n_{a}(E) \delta=\frac{1}{\pi} \frac{E}{E_{T}}\left(\alpha-\beta \ln \frac{E}{E_{T}}\right), \\
\alpha=\frac{W^{2}}{A}\left[C_{1}+C_{2}\left(\kappa+\ln \frac{2 \pi^{2}}{W / \sqrt{A}}\right)\right], \\
\kappa=-\frac{1}{2}-\frac{W^{2}}{3} \ln 2-\frac{6}{\pi^{2}} \zeta^{\prime}(2) \approx-0.854235,
\end{gathered}
$$

and $E_{\mathrm{T}}=M \delta /(4 \pi)$ is the Thouless energy. ${ }^{3}$ Besides the term proportional to the energy (which was also predicted in Refs. 3 and 4), there is an additional logarithmic factor. Moreover, in contrast to the above mentioned references the coefficient $\alpha$ depends on $W, a$, and $d$. However, $\beta$ depends only on $W / a$. Note that $W=a$ is a special case corresponding to the system studied by de Gennes and Saint-James. ${ }^{2}$ Then, $C_{2}$ $=0$ and no logarithmic factor appears in the DOS and the result is the same as in Ref. 6.

It is interesting to see the $W \ll a$ limit for which one would expect that $P(s)$ has a universal limiting form, namely it is only a function of $W^{2} / A$. In this case, the electron has enough time to explore the whole available phase space be- 


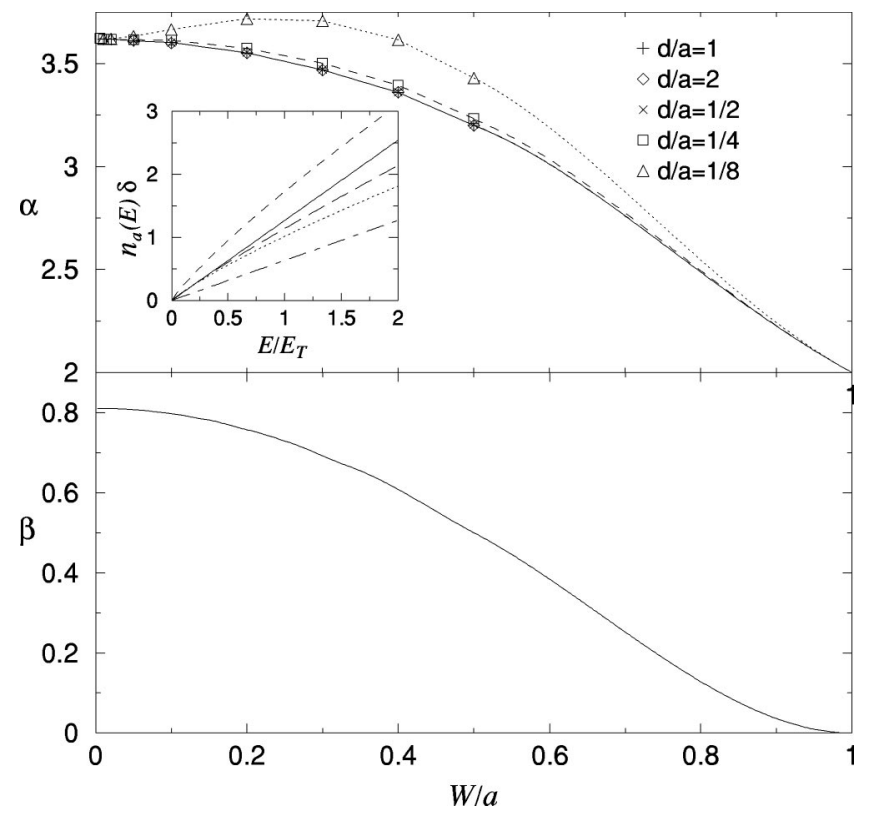

FIG. 4. Top panel: $\alpha$ as a function of $W / a$ for $d / a$ $=1,2,1 / 2,1 / 4,1 / 8$. Bottom panel: $\beta$ as a function of $W / a$ obtained from Eq. (8d) using Eqs. (6b) and (7) for $C_{2}$. The inset of the top panel shows the asymptotic DOS $n_{a}(E)$ (in units of $1 / \delta$ ) for $d$ $=a, W / a=1,0.5,0.1,0.01$ (dot-dashed, dotted, long-dashed, and dashed lines, respectively) and the result from Ref. 3 (solid line).

fore escaping (even in an integrable billiard) and $P(s)$ looses any detailed dependence on the geometry of the billiard. Therefore, two billiards with the same $W^{2} / A$ but different aspect ratio $(d / a)$ should have the same path length distribution for small enough $W$. From numerical calculations we found that for $W \rightarrow 0, \alpha$ converges to a value $\alpha \approx 3.62$ independent of the aspect ratio, i.e., it becomes a universal con- stant. Similarly we find that when $W \rightarrow 0, \beta$ also tends to a universal constant $\beta \rightarrow 8 / \pi^{2}$. These results are demonstrated in Fig. 4. In the inset of the top panel of Fig. 4 the asymptotic DOS $n_{a}(E)$ obtained from Eq. (8b) is plotted for different values of $W$ along with the result from Ref. 3 for the sake of comparison. Our results are in the same order of magnitude as that found in Melsen et al. ${ }^{3}$ and Ihra et al. ${ }^{4}$ However, from our analysis it turns out that the functional form of the asymptotic DOS is not just a linear function but involves a logarithmic factor.

In conclusion, we have shown that exact quantum mechanical calculations for the integrated DOS of rectangular Andreev billiards agrees well (for the whole energy range below the gap) with that obtained from the Bohr-Sommerfeld approximation provided the energy dependent phase shift is taken into account. From the exact analytic form of the asymptote of the integrated return probability, we predict a new, logarithmic contribution to the DOS on the scale of the Thouless energy. In contrast to earlier results, we show that the DOS at this energy range explicitly depends on $W, a$, and $d$ but it has a universal limiting form for small enough $W$. We also investigated the case when the superconductor is placed anywhere at the side of the rectangle and found that the logarithmic contribution in the DOS is generic for rectangular Andreev billiards.

One of us (J.Cs.) gratefully acknowledges very helpful discussions with C. W. J. Beenakker. This work was supported in part by the EU's Human Potential Program under Contract No. HPRN-CT-2000-00144, the Hungarian-British Intergovernmental Agreement on Cooperation in Education, Culture, and Science and Technology, and the Hungarian Science Foundation OTKA TO34832. One of us (Z.K.) thanks the Hungarian Academy of Sciences for its financial support as a János Bolyai Research Fellowship.
*Electronic address: kor@complex.elte.hu

${ }^{1}$ I. Kosztin, D. L. Maslov, and P. M. Goldbart, Phys. Rev. Lett. 75, 1735 (1995); A. Altland and M. R. Zirnbauer, ibid. 76, 3420 (1996); G. B. Lesovik, A. L. Fauchère, and G. Blatter, Phys. Rev. B 55, 3146 (1997); A. Lodder and Y. V. Nazarov, ibid. 58, 5783 (1998); H. Schomerus and C. W. J. Beenakker, Phys. Rev. Lett. 82, 2951 (1999); W. Ihra and K. Richter, Physica E (Amsterdam) 9, 362 (2001).

${ }^{2}$ P. G. de Gennes and D. Saint-James, Phys. Lett. 4, 151 (1963).

${ }^{3}$ J. A. Melsen, P. W. Brouwer, K. M. Frahm, and C. W. J. Beenakker, Europhys. Lett. 35, 7 (1996); J. A. Melsen, P. W. Brouwer, K. M. Frahm, and C. W. J. Beenakker, Phys. Scr., T 69, 223 (1997).

${ }^{4}$ W. Ihra, M. Leadbeater, J. L. Vega, and K. Richter, Eur. Phys. J. B 21, 425 (2001).
${ }^{5}$ J. Cserti, A. Kormányos, Z. Kaufmann, J. Koltai, and C. J. Lambert, Phys. Rev. Lett. 89, 057001 (2002).

${ }^{6}$ J. Cserti, A. Bodor, J. Koltai, G. Vattay, Phys. Rev. B 66, 064528 (2002).

${ }^{7}$ P. G. de Gennes, Superconductivity of Metals and Alloys (Benjamin, New York, 1996).

${ }^{8}$ C. J. Lambert and R. Raimondi, J. Phys.: Condens. Matter 10, 901 (1998); O. Šipr and B. L. Györffy, ibid. 8, 169 (1996): J. Low Temp. Phys. 106, 315 (1997).

${ }^{9}$ In Refs. 3 and 4 the geometry of $\mathrm{AB}$ can be obtained by cutting our $\mathrm{AB}$ at its symmetry axis. Our results are irrelevant to this geometrical difference.

${ }^{10}$ N. A. Mortensen, A.-P. Jauho, K. Flensberg, and H. Schomerus, Phys. Rev. B 60, 13762 (1999). 